SYNLETT Spotlight 12

This feature focuses on a reagent chosen by a postgraduate, highlighting the uses and preparation of the reagent in current research

\section{Scandium Triflate}

Compiled by Deborah Longbottom

Department of Chemistry, University of Cambridge, Lensfield Road, Cambridge, CB2 1EW, UK.

Deborah Longbottom studied chemistry at the University of Durham where she received her B.Sc. in 1997. She then worked for one year for GlaxoWellcome Plc. in Stevenage, Herts and is currently working on her Ph.D. thesis under the supervision of Professor Steven V. Ley.

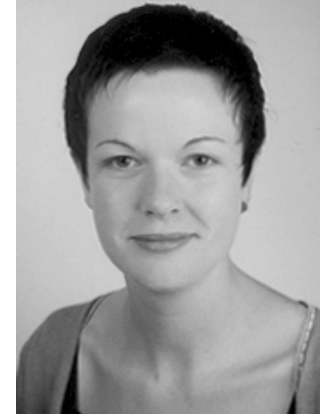

Scandium triflate (1) is a new type of Lewis acid, differing from typical Lewis acids such as $\mathrm{AlCl}_{3}, \mathrm{BF}_{3}$ and $\mathrm{TiCl}_{4}{ }^{1}$ It is not decomposed or deactivated in the prescence of water and will work as a catalyst where other Lewis acids are required in stoichiometric quantities. This is exemplified by its catalytic activity in the aldol reaction of silyl enol ethers with aldehydes and acetals, ${ }^{2}$ Michael reactions, ${ }^{2}$ Friedel-Crafts acylations $^{3}$ and Diels-Alder reactions ${ }^{4}$ among others. Moreover, many nitrogen containing compounds can be activated by a catalytic amount of $\mathrm{Sc}(\mathrm{OTf})_{3}$ in both organic and aque- ous solvents. ${ }^{5}$ Scandium triflate can be recovered almost quantitatively after the desired reaction and can be recycled in subsequent reactions without loss of activity. ${ }^{1}$

Preparation: Scandium triflate is commercially available but can also be prepared from the corresponding oxide $\left(\mathrm{Sc}_{2} \mathrm{O}_{3}\right)$ and aqueous trifluoromethanesulfonic acid $(\mathrm{TfOH}){ }^{4}$

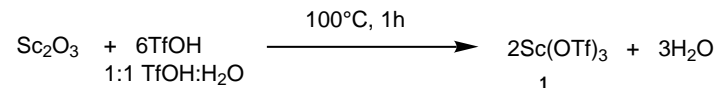

\title{
Abstracts
}

A) Friedel-Crafts alkyl- and acylation reactions usually require a stoichiometric amount of Lewis acid to facilitate complete reaction. However, a catalytic amount of $\mathrm{Sc}(\mathrm{OTf})_{3}$ is sufficient to facilitate the same transformation.,

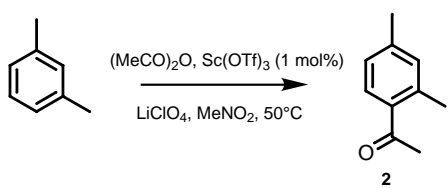

B) $\mathrm{Sc}(\mathrm{OTf})_{3}$ catalysed three-component couplings can be carried out affording amino esters 3 and $\gamma$-acyl- $\delta$-lactam derivatives $\mathbf{4}$ stereoselectively in high yields. ${ }^{7}$ This is a powerful tool for the preparation of libraries of $\delta$-lactam derivatives. Similar fourcomponent couplings have also been carried out. ${ }^{7}$

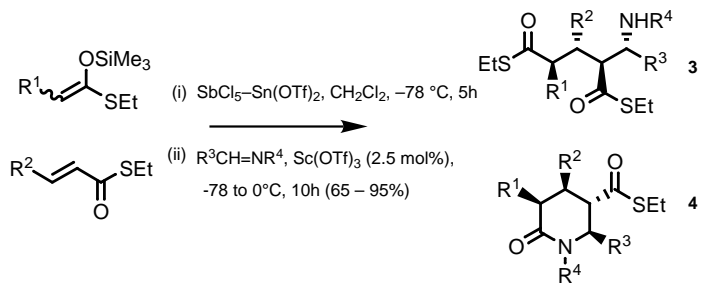

C) $\mathrm{Sc}(\mathrm{OTf})_{3}$ is a useful Lewis acid catalyst for acylation of alcohols with acid anhydrides and esterification between alcohols and carboxylic acids. ${ }^{8}$

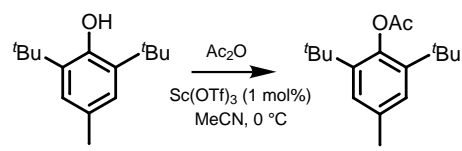

D) Allylation reactions of carbonyl compounds with tetraallyltin (6) occur smoothly under the influence of a catalytic amount of $\mathrm{Sc}(\mathrm{OTf})_{3}{ }^{9}$ Three component reactions of aldehydes, amines and allyltributyltin (8) also proceed smoothly. ${ }^{10}$ No direct reaction with aldehyde is observed.

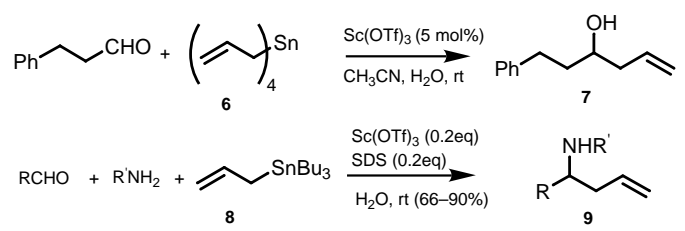

\section{References and Notes}

(1) Kobayashi, S.; Eur. J. Org. Chem. 1999, 15.

(2) Kobayashi, S.; Hachiya, I.; Ishitani, H.; Araki, M.; Synlett 1993, 472

(3) Kawada, A.; Mitamura, S.; Kobayashi, S.; J. Chem. Soc., Chem. Commun. 1996, 183.

(4) Kobayashi, S.; Hachiya I.; Araki, M.; Ishitani, H.; Tetrahedron Lett. 1993, 34, 3755.

(5) Kobayashi, S.; Ishitani, H.; Nagayama, S.; Synthesis 1995, 1195.
(6) Tsuchimoto, T.; Tobita, K.; Hiyama, T.; Fukuzawa, S.; J. Org. Chem. 1997, 6997.

(7) Kobayashi, S.; Akiyama, R.; Moriwaki, M.; Tetrahedron Lett. 1997, 38, 4819.

(8) Ishihara, K.; Kubota, M.; Kurihara, H.; Yamamoto, H.; J. Am. Chem. Soc. 1995, 117, 4413

(9) Hachiya, I.; Kobayashi, S.; J. Org. Chem. 1993, 58, 6958.

(10) Akiyama, T.; Iwai, J.; Synlett 1998, 273.

Article Identifier:

1437-2096,E;1999,0,12,2023,2023,ftx,en;V01399ST.pdf 